\section{Variability in protein and mineral content of pasture and turf grasses}

\author{
Elaine M. Allen', William Meyer ${ }^{2}$ and Sarah L. Ralston' \\ Departments of Animal Science ${ }^{1}$ and Plant Science ${ }^{2}$, Rutgers University, \\ New Brunswick, USA
}

\section{Introduction}

Protein, energy and mineral intake dramatically impact bone growth and development in young horses (Jeffcott 1991). Concentrations of non-structural carbohydrates (NSC), water soluble sugars (WSS fraction: glucose, fructose, sucrose and fructans) can fluctuate significantly between forage species and with changes in environment (Allen et al. 2005). However diurnal and seasonal variations in protein and mineral content in common pasture grass mixes and newer turf grass species (Meyer 2004) have not been documented. It was hypothesized that common pasture mixes used in northeastern United States (i.e.: timothy, bluegrass, fescue, orchard grass and clover) and new turf grass species being considered for use in horse pastures would have significant seasonal and possibly diurnal fluctuations in protein and minerals.

\section{Materials and Methods}

Representative samples (at least ten sites per pasture at each collection for a total 500 grams wet weight) of pastures actively grazed by horses were taken from four New Jersey farms with different soil types: Sandy marl (SM), marl, heavy loam/clay/marl (HLCM), sand and loam on farms in Monmouth County (eastern shore/central region) and clay in Mercer County in the western part of the state. Samples were collected in May, 2004 (late spring) and at 4 week intervals from August-November, 2004 (early to late fall) at 7-9AM and again on the same day at 4-7PM. Previous night temperature, current weather conditions, height and maturity of the samples were recorded, as was whether or not the pasture had been fertilized. Ground temperature was not recorded. Additional samples were taken after the first hard overnight freeze $(11 / 13 / 04)$.

The same sampling protocol was used for pure cultivars of four turf grasses at the Adelphia Turfgrass Research Center (loam soil in eastern Monmouth County): Colonial Bentgrass (Tiger II), (Agrostis tenuis L), Texas X Kentucky bluegrass hybrid (Thermal) (Poa aracinifera L. X Poa pratensis L.), strong creeping fescue (SCFescue), (Festuca rubra subsp.rubra L.) and hard fescue (HFescue) ) Festuca brevipila (Hack) Krajina). The latter two are fescue varieties with low or no endophyte. In addition a non-turf warm season grass, crabgrass (CG) (Digitaria sanguiinalis( L.) Scop), was sampled. Five to six pure cultivars of each species were sampled at each collection. The crabgrass samples were going to seed and into dormancy when collected.
Samples were placed in dry ice immediately and stored frozen pending freeze-drying. Freeze-dried samples were submitted for wet chemistry analysis of crude protein, non-structural soluble carbohydrates (NSC), acid detergent fiber (ADF), calcium (Ca), phosphorus $(P)$, potassium $(K)$, magnesium $(\mathrm{Mg})$, copper (Cu), zinc (ZN) and iron (FE) (Equi-Analytical Laboratories, Ithaca, NY).

Pearson's Correlation analysis was used to determine significant interactions. Linear regression analysis was used to test relationships between linear variables (ie: nutrient contents, temperature, date). Effects of discrete factors such as farm, collection site or grass type were assessed using ANOVA and LSD (Statistix 8.0, Ocala, FL).

\section{Results}

Protein and mineral content did not show diurnal variations. There were nutrient differences $(P<0.05)$ among turf grass species (Table 1) and among pastures (Table 2). Mature plants (full seed head) had lower $\mathrm{Zn}(\mathrm{P}<0.05)$ and higher ADF $(P<0.05)$ than immature, pre seed head or emerging seed head plants. There were seasonal variations $(P<0.05)$ in some of the nutrients (Table 3 ). Protein content of the gras-

Table 1a Turf Grass DM macromineral content. All cultivars were grown on the same soil type and sampled at the same times. Values are means $\pm \mathrm{SE}$.

\begin{tabular}{|l|l|l|l|l|l|}
\hline Grass Type & $\mathbf{N}$ & $\% \mathrm{Ca}$ & $\% \mathrm{P}$ & $\% \mathrm{~K}$ & $\% \mathrm{Mg}$ \\
\hline Thermal & 6 & $.36 \pm .06^{\mathrm{a}}$ & $.30 \pm .01^{\mathrm{b}}$ & $2.38 \pm .1^{\mathrm{ab}}$ & $.21 \pm .01^{\mathrm{b}}$ \\
\hline SCFescue & 6 & $.37 \pm .02^{\mathrm{a}}$ & $.37 \pm .02^{\mathrm{a}}$ & $2.66 \pm .13^{\mathrm{a}}$ & $.20 \pm .02^{\mathrm{bc}}$ \\
\hline HFescue & 6 & $.25 \pm .02^{\mathrm{b}}$ & $.28 \pm .10^{\mathrm{b}}$ & $2.13 \pm .15^{\mathrm{b}}$ & $.13 \pm .01^{\mathrm{c}}$ \\
\hline Tiger II & 5 & $.35 \pm .02^{\mathrm{a}}$ & $.31 \pm .03^{\mathrm{ab}}$ & $2.39 \pm .22^{\mathrm{ab}}$ & $.26 \pm .03^{\mathrm{b}}$ \\
\hline CB & 4 & $.30 \pm .03^{\mathrm{ab}}$ & $.32 \pm .03^{\mathrm{ab}}$ & $2.10 \pm .28^{\mathrm{b}}$ & $.60 \pm .14^{\mathrm{a}}$ \\
\hline
\end{tabular}

Table 1b Turf Grass DM micromineral content.

\begin{tabular}{|l|l|l|l|l|l|}
\hline Grass Type & $\mathbf{N}$ & \multicolumn{1}{|c|}{ Cu ppm } & Fe ppm & Mn ppm & Zn ppm \\
\hline Thermal & 6 & $11 \pm 1^{\mathrm{ab}}$ & $635 \pm 114^{\mathrm{b}}$ & $63 \pm 9^{\mathrm{a}}$ & $26 \pm 2$ \\
\hline SCFescue & 6 & $9 \pm .4^{\mathrm{b}}$ & $771 \pm 131^{\mathrm{b}}$ & $52 \pm 6^{\mathrm{ab}}$ & $21 \pm 1$ \\
\hline HFescue & 6 & $13 \pm 1^{\mathrm{a}}$ & $1680 \pm 345^{\mathrm{a}}$ & $49 \pm 5^{\mathrm{ab}}$ & $25 \pm 3$ \\
\hline Tiger II & 5 & $10 \pm 1^{\mathrm{ab}}$ & $1032 \pm 260^{\mathrm{ab}}$ & $65 \pm 13^{\mathrm{a}}$ & $25 \pm 6$ \\
\hline CB & 4 & $11 \pm 1^{\mathrm{ab}}$ & $559 \pm 515^{\mathrm{b}}$ & $33 \pm 4^{\mathrm{b}}$ & $28 \pm 3$ \\
\hline
\end{tabular}

Table 1c Turf Grass DM Protein, starch, NSC and fiber content

\begin{tabular}{|l|l|l|l|l|l|}
\hline Grass Type & $\mathbf{N}$ & Protein & Starch & NSC & ADF \\
\hline Thermal & 6 & $20.8 \pm 2.2$ & $1.6 \pm 0.1^{\mathrm{b}}$ & $14.5 \pm 2.0^{\mathrm{a}}$ & $29.0 \pm 1.4$ \\
\hline SCFescue & 6 & $21.5 \pm 1.2$ & $1.7 \pm .5^{\mathrm{b}}$ & $10.4 \pm 1.8^{\mathrm{ab}}$ & $29.4 \pm 1.2$ \\
\hline HFescue & 6 & $19.0 \pm 1.6$ & $1.1 \pm 0.2^{\mathrm{b}}$ & $9.0 \pm 2.1^{\mathrm{b}}$ & $32.3 \pm 2.0$ \\
\hline Tiger II & 5 & $20.4 \pm 1.6$ & $1.7 \pm 0.2^{\mathrm{b}}$ & $9.9 \pm 1.9^{\mathrm{a}, \mathrm{b}}$ & $36.1 \pm 5.8$ \\
\hline CB & 4 & $13.7 \pm 1.0$ & $6.6 \pm 0.8^{\mathrm{a}}$ & $13.3 \pm 2.0^{\mathrm{a}, \mathrm{b}}$ & $34.6 \pm 3.4$ \\
\hline
\end{tabular}

Table 2a Pasture mix DM macromineral content. Values are means \pm SE.

\begin{tabular}{|l|l|l|l|l|l|l|}
\hline Farm & $\begin{array}{l}\text { Pasture } \\
\text { Soil type }\end{array}$ & $\mathbf{N}$ & $\mathbf{C a} \%$ & $\mathbf{P} \%$ & $\mathbf{K} \%$ & $\mathbf{M g} \%$ \\
\hline $1 \mathrm{a}$ & Loam & 5 & $.61 \pm .04^{\mathrm{b}}$ & $.37 \pm .02^{\mathrm{cd}}$ & $2.22 \pm .11^{\mathrm{b}}$ & $.33 \pm .03^{\mathrm{a}}$ \\
\hline $1 \mathrm{~b}$ & Loam & 5 & $.48 \pm .03^{\mathrm{cd}}$ & $.53 \pm .02^{\mathrm{a}}$ & $1.72 \pm .06^{\mathrm{d}}$ & $.24 \pm .01^{\mathrm{cd}}$ \\
\hline $1 \mathrm{c}$ & Loam & 5 & $.51 \pm .03^{\mathrm{c}}$ & $.47 \pm .02^{\mathrm{ab}}$ & $2.18 \pm .09^{\mathrm{b}}$ & $.30 \pm .01^{\mathrm{ab}}$ \\
\hline 2 & Sand & 4 & $.41 \pm .04^{\mathrm{de}}$ & $.33 \pm .01^{\mathrm{d}}$ & $1.89 \pm .05^{\mathrm{cd}}$ & $.22 \pm .01^{\mathrm{d}}$ \\
\hline 3 & $\mathrm{Clay}$ & 5 & $.74 \pm .03^{\mathrm{a}}$ & $.34 \pm .01^{\mathrm{d}}$ & $2.14 \pm .13^{\mathrm{bc}}$ & $.28 \pm .01^{\mathrm{abc}}$ \\
\hline $4 \mathrm{a}$ & $\mathrm{SM}$ & 4 & $.32 \pm .02^{\mathrm{e}}$ & $.48 \pm .03^{\mathrm{ab}}$ & $2.62 \pm .12^{\mathrm{a}}$ & $.29 \pm .01^{\mathrm{ab}}$ \\
\hline $4 \mathrm{~b}$ & Marl & 4 & $.43 \pm .04^{\mathrm{cd}}$ & $.38 \pm .03^{\mathrm{cd}}$ & $2.10 \pm .10^{\mathrm{bc}}$ & $.22 \pm .01^{\mathrm{d}}$ \\
\hline $4 \mathrm{c}$ & $\mathrm{LCM}$ & 4 & $.45 \pm .02^{\mathrm{cd}}$ & $.42 \pm .03^{\mathrm{bc}}$ & $2.59 \pm .12^{\mathrm{a}}$ & $.25 \pm .02^{\mathrm{cd}}$ \\
\hline $4 \mathrm{~d}$ & $\mathrm{LCM}$ & 4 & $.42 \pm .02^{\mathrm{cd}}$ & $.42 \pm .03^{\mathrm{bc}}$ & $2.62 \pm .07^{\mathrm{a}}$ & $.24 \pm .02^{\mathrm{cd}}$ \\
\hline
\end{tabular}


Table 2b Pasture mix DM micromineral content.

\begin{tabular}{|l|l|l|l|l|l|l|}
\hline Farm & $\begin{array}{l}\text { Pasture } \\
\text { Soil type }\end{array}$ & $\mathbf{N}$ & Cu ppm & Fe ppm & Mn ppm & Zn ppm \\
\hline $1 a$ & Loam & 5 & $10 \pm .4$ & $1393 \pm 271^{\text {abc }}$ & $62 \pm .5^{b c}$ & $31 \pm 2$ \\
\hline $1 b$ & Loam & 5 & $17 \pm 1$ & $2575 \pm 1563^{a}$ & $46 \pm 10^{c d}$ & $34 \pm 5$ \\
\hline $1 c$ & Loam & 5 & $10 \pm 2$ & $473 \pm 135^{c}$ & $25 \pm 3^{d}$ & $28 \pm 2$ \\
\hline 2 & Sand & 4 & $9 \pm 1$ & $162 \pm 5^{c}$ & $98 \pm 12^{a}$ & $31 \pm 3$ \\
\hline 3 & Clay & 5 & $9 \pm .5$ & $569 \pm 66^{c}$ & $82 \pm 15^{\text {ab }}$ & $(63 \pm 21)^{*}$ \\
\hline $4 a$ & SM & 4 & $10 \pm 1$ & $664 \pm 130^{\text {bc }}$ & $30 \pm 6^{d}$ & $31 \pm 2$ \\
\hline $4 b$ & Marl & 4 & $9 \pm 1$ & $725 \pm 118^{\text {bc }}$ & $95 \pm 22^{a}$ & $29 \pm 2$ \\
\hline $4 c$ & LCM & 4 & $9 \pm 1$ & $1415 \pm 186^{\text {abc }}$ & $33 \pm 2^{c d}$ & $33 \pm 2$ \\
\hline $4 d$ & LCM & 4 & $11 \pm 1$ & $2407 \pm 228^{\text {ab }}$ & $46 \pm 4^{c d}$ & $35 \pm 4$ \\
\hline
\end{tabular}

Table 2c Pasture mix DM protein, starch, NSC and ADF content.

\begin{tabular}{|l|l|l|l|l|l|l|}
\hline Farm & $\begin{array}{l}\text { Pasture } \\
\text { Soil type }\end{array}$ & $\mathbf{N}$ & Protein \% & Starch\% & NSC\% & ADF\% \\
\hline $1 \mathrm{a}$ & Loam & 5 & $23.8 \pm 1.1^{\mathrm{a}}$ & $3.1 \pm .6^{\mathrm{ab}}$ & $10.3 \pm 1.3^{\mathrm{b}}$ & $28.6 \pm .9^{\mathrm{bc}}$ \\
\hline $1 \mathrm{~b}$ & Loam & 5 & $11.1 \pm 1.3^{\mathrm{e}}$ & $5.7 \pm 1.3^{\mathrm{a}}$ & $13.8 \pm 1.9^{\mathrm{ab}}$ & $31.3 \pm 1.1^{\mathrm{a}}$ \\
\hline $1 \mathrm{c}$ & Loam & 5 & $20.2 \pm 1.1^{\mathrm{abc}}$ & $2.7 \pm .5^{\mathrm{b}}$ & $12.3 \pm .9^{\mathrm{ab}}$ & $30.3 \pm .9^{\mathrm{ab}}$ \\
\hline 2 & Sand & 4 & $14.3 \pm 1.3^{\mathrm{de}}$ & $5.6 \pm .5^{\mathrm{a}}$ & $14.9 \pm .7^{\mathrm{ab}}$ & $29.8 \pm .6^{\mathrm{ab}}$ \\
\hline 3 & Clay & 5 & $19.8 \pm 1.0^{\mathrm{bc}}$ & $2.5 \pm .5^{\mathrm{b}}$ & $15.5 \pm 2.6^{\mathrm{ab}}$ & $28.2 \pm 1.1^{\mathrm{bc}}$ \\
\hline $4 \mathrm{a}$ & SM & 4 & $17.1 \pm 2.8^{\mathrm{cd}}$ & $4.4 \pm 2.2^{\mathrm{ab}}$ & $15.8 \pm 2.5^{\mathrm{ab}}$ & $28.3 \pm .6^{\mathrm{bc}}$ \\
\hline $4 \mathrm{~b}$ & Marl & 4 & $16.5 \pm 1.0^{\mathrm{cd}}$ & $2.1 \pm .8^{\mathrm{b}}$ & $13.6 \pm 2.6^{\mathrm{ab}}$ & $31.3 \pm 1.1^{\mathrm{a}}$ \\
\hline $4 \mathrm{c}$ & LCM & 4 & $23.3 \pm 1.0^{\mathrm{ab}}$ & $3.0 \pm .8^{\mathrm{ab}}$ & $17.2 \pm 1.9^{\mathrm{a}}$ & $26.5 \pm .4^{\mathrm{c}}$ \\
\hline $4 \mathrm{~d}$ & LCM & 4 & $21.4 \pm 1.0^{\mathrm{ab}}$ & $3.3 \pm 1.2^{\mathrm{ab}}$ & $16.3 \pm 2.6^{\mathrm{a}}$ & $26.4 \pm 1.2^{\mathrm{c}}$ \\
\hline
\end{tabular}

$a, b, c, d$ Nutrient means with different superscripts differ $(P<0.05)$ between pastures.

* Zinc content of 2 samples unusually high (77 and 142 ppm)-probable contamination.

Table 3 Seasonal variation in DM nutrient content of pasture mixes. Values are means \pm SE.

\begin{tabular}{|c|c|c|c|c|c|}
\hline Date & May & August & September & October & November* \\
\hline Protein \% & $19.9 \pm 0.7$ & $17.6 \pm 1.4$ & $17.5 \pm 2.6$ & $18.7 \pm 1.7$ & $20.7 \pm 2.1$ \\
\hline Starch \% & $2.0 \pm 0.4^{b c}$ & $4.8 \pm 0.6^{\circ}$ & $4.7 \pm 0.6^{\circ}$ & $3.5 \pm 0.6^{\mathrm{ab}}$ & $0.78 \pm 0.1^{\circ}$ \\
\hline NSC $\%$ & $13.2 \pm 0.6^{b}$ & $13.9 \pm 1.1^{b}$ & $11.2 \pm 0.6^{b}$ & $13.8 \pm 1.3^{b}$ & $20.9 \pm 1.2^{\circ}$ \\
\hline Ca \% & \begin{tabular}{|l}
$.53 \pm .04^{\mathrm{ab}}$ \\
\end{tabular} & $.43 \pm .03^{b}$ & $.50 \pm .03^{b}$ & $.65 \pm .05^{\circ}$ & $.46 \pm .07^{b}$ \\
\hline $\mathrm{P} \%$ & $.44 \pm .03^{\circ}$ & $.43 \pm .02^{\circ}$ & $.48 \pm .03^{\circ}$ & $.34 \pm .01^{\mathrm{b}}$ & $.35 \pm .02^{b}$ \\
\hline $\mathrm{Mg} \%$ & $.25 \pm .02^{b}$ & $.26 \pm .01^{b}$ & $.32 \pm .02^{A}$ & $.27 \pm .02^{\mathrm{ab}}$ & $.21 \pm .02^{\mathrm{ob}}$ \\
\hline $\mathrm{K} \%$ & $2.2 \pm 0.1^{b}$ & $2.3 \pm 0.1^{b}$ & $2.0 \pm 0.1^{b}$ & $2.0 \pm 0.04^{b}$ & $2.6 \pm 0.2^{\circ}$ \\
\hline Cu ppm & $17.3 \pm 5.5^{a}$ & $9.3 \pm 0.4^{b}$ & $9.8 \pm 0.6^{b}$ & $9.8 \pm 0.5^{b}$ & $7.2 \pm 0.4^{b}$ \\
\hline Fe ppm & $2015 \pm 1124^{\circ}$ & $998 \pm 249^{\mathrm{ab}}$ & $1184 \pm 218^{a b}$ & $469 \pm 101^{b}$ & $1185 \pm 305^{\mathrm{ob}}$ \\
\hline Mn ppm & $51 \pm 8^{b}$ & $53 \pm 9^{b}$ & $44 \pm 9^{b}$ & $96 \pm 13^{\circ}$ & $45 \pm 10^{b}$ \\
\hline Zn ppm & $32 \pm 3^{\circ}$ & $31 \pm 1^{a}$ & $33 \pm 3^{\circ}$ & $41 \pm 7^{\circ}$ & $51 \pm 23^{\circ}$ \\
\hline
\end{tabular}

$a b, c, d=$ nutrient means with different superscripts differ $P<0.05$ over time.

*Samples taken after an overnight freeze.

** Zinc content of samples from farm 3 unusually high (77 and 142 ppm)-probable contamination.

ses was correlated $(\mathrm{P}<0.05)$ with sugar, $\mathrm{K}, \mathrm{Mn}, \mathrm{Ca}$, and $\mathrm{Zn}$ yet was inversely correlated $(P<0.05)$ with starch, NDF, ADF, previous night temperature, and the temperature when sample was taken.

\section{Discussion}

Though the water-soluble sugars in these forages increased $(\mathrm{P}<0.05)$ over the course of a day or after an overnight freeze (Watts and Chatterton 2004), protein and minerals did not vary $(P>0.05)$ under the same circumstances, and starch actually decreased after an overnight freeze. Only NSC, which consists of water-soluble sugars plus starch (Allen et al. 2005), had significant variability over the course of a day or after a freeze. This is predominantly of concern to horses that are prone to laminitis.

Unexpectedly copper and iron did not differ $(\mathrm{P}<0.05)$ between farms, despite differences in soil types and grass species. However there were differences in other minerals, even between pastures on the same farm on the same soil type. For example on farm 1 pasture la was adjacent to a heavy fertilized agricultural field and lightly grazed, whereas $1 \mathrm{~b}$ was farther downhill, overgrazed (grasses $<4 \mathrm{~cm}$ ) with $<10 \%$ clover and the adjacent 1c was virtually unused (grasses $>15$ $\mathrm{cm}$ ) with $>20 \%$ clover content. The calcium/phosphorus ratio was reversed in the hard fescue turf grass and on 2 pastures: pasture $1 \mathrm{~b}$ and pasture $4 \mathrm{a}$, which had very poor soil (sandy marl), was at the top of a hill and also had $<10 \%$ clover. All of the pastures on farms 1, 2 and 4 had been fertilized with calcium within the past year.

The protein content of all forages tested except crabgrass would meet or exceed the recommendations for both adult and growing horses (NRC 1989). However the calcium and phosphorus content of all the turf grasses were deficient relative to the needs of rapidly growing foals 4 , and only Tiger II and pasture 3 had adequate zinc. Use of pastures 1c, $4 \mathrm{a}$ or any of the turf grasses as the primary source of nutrition for growing horses would probably result in a high incidence of developmental orthopedic disease (Jeffcott 1991) if additional minerals were not supplemented. All pastures except 1c contained adequate minerals to meet the needs of mature maintenance horses with free access to water and salt. There were significant seasonal variations in the mineral content of pasture mixes, with the month of August, after a period of very hot, dry weather showing the least desirable mineral content.

\section{Conclusions}

It should not be assumed that adequate calcium is provided by commonly used pasture mixes under certain soil/growing conditions. Some turf grass species selected for their hardiness may be appropriate for use in pastures for adult animals but may not be appropriate for breeding farms unless overseeded with a palatable legume or the horses are fed a mineral supplement.

\section{References}

Allen E. A., Meyer W., Ralston S. L. and Watts K. A. (2005): Variation in soluble sugar content of pasture and turf grasses. Proc Equine Science Society Symposium, Tucson, 321-323

Jeffcott L. B. (1991): Osteochondrosis in the horse - searching for the key to pathogenesis. Equine Vet J 23, 331-338

National Research Council (1989): Nutrient Requirements of Horses. National Academy Press, Washington, DC Meyer W. (2004): personal communication

Watts K. A. and Chatterton N. J. (2004): A review of factors affecting carbohydrate levels in forage. J Equine Vet Sience 24, 84-86

The authors acknowledge Kathryn Watts, Rocky Mountain Consulting, Inc, for her input and advice on this project.

\section{E. M. Allen}

Departments of Animal Science \& Plant Science

Rutgers University

New Brunswick, NJ

Ralston@aesop.rutgers.edu 